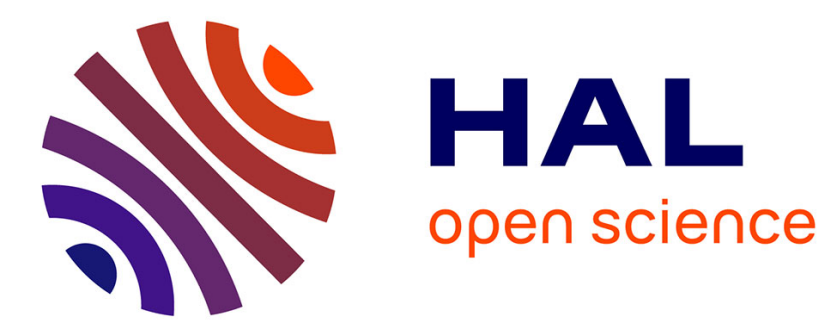

\title{
Issues in Modeling Cardiac Optical Mapping Measurements
}

Gwladys Ravon, Yves Coudière, Angelo Iollo, Oliver Bernus, Richard D. Walton

\section{- To cite this version:}

Gwladys Ravon, Yves Coudière, Angelo Iollo, Oliver Bernus, Richard D. Walton. Issues in Modeling Cardiac Optical Mapping Measurements. Functional Imaging and Modeling of the Heart, Jun 2015, Maastricht, Netherlands. 10.1007/978-3-319-20309-6_52 . hal-01214073

\section{HAL Id: hal-01214073 https://hal.science/hal-01214073}

Submitted on 13 Nov 2015

HAL is a multi-disciplinary open access archive for the deposit and dissemination of scientific research documents, whether they are published or not. The documents may come from teaching and research institutions in France or abroad, or from public or private research centers.
L'archive ouverte pluridisciplinaire HAL, est destinée au dépôt et à la diffusion de documents scientifiques de niveau recherche, publiés ou non, émanant des établissements d'enseignement et de recherche français ou étrangers, des laboratoires publics ou privés. 


\title{
Issues in Modeling Cardiac Optical Mapping Measurements
}

\author{
Gwladys Ravon ${ }^{1,2,3}$, Yves Coudière ${ }^{1,2,3}$, Angelo Iollo ${ }^{1,3}$, Oliver Bernus ${ }^{2}$, \\ Richard D Walton ${ }^{2}$ \\ 1 Inria Bordeaux Sud-Ouest, Bordeaux, France \\ 2 LIRYC, L'Institut de rythmologie et modélisation cardiaque, Université de \\ Bordeaux, F33000, Bordeaux \\ 3 Institut de Mathématiques de Bordeaux, Bordeaux, France
}

\begin{abstract}
Optical mapping allows to visualize cardiac action potentials (AP) on cardiac tissue surfaces by fluorescence using voltage-sensitive dyes. So far, the surface measurements are directly related to surface AP. In a previous study was developed a method to reconstruct threedimensional depolarization front: the main idea was to solve an inverse problem using the experimental measures on the surfaces. Although the method was very accurate on in silico data, it showed difficulties to recover real optical mapping measurements. Here we describe the different directions we followed to improve the results.
\end{abstract}

Keywords: mathematical modeling, optical mapping, inverse problem

\section{Introduction}

Optical mapping is an important tool for the understanding of cardiac arrhythmias [1]. It provides surface optical recordings that are linked to surface AP [5]. Although the photons are known to interact with the tissue up to a few $\mathrm{mm}$ in depth, it remains very challenging to actually retrieve 3D information from optical recordings. In [2], Khait et al derive a formula to determine the depth of some fixed electrical sources in a phantom. Instead, we propose to solve an inverse problem, so as to recover more accurate and complete information on the electrical sources. The approach is also expected to apply to more general experimental conditions. In a previous study we presented a first attempt to obtain a complete 3D reconstruction following this approach. Although we obtained excellent agreement between the actual location of the source and the location found by the inverse method, on in silico data, the results on the experimental data provided by the authors of [2] were disappointing. Actually, we observed a large mismatch between the experimental measures and the measures computed from the optical mapping model and the known experimental locations. Here we explore the model and its optical parameters as a cause of this mismatch.

\section{Optical mapping}

For optical mapping of AP, fluorescent voltage-sensitive dyes that attach to the cells' membrane are injected into a slab of tissue. The tissue is put in a bath, 
and cameras and lights are placed on both sides of the preparation (epicardium and endocardium, fig. 1). A filter is placed in front of each camera, that allows to choose the wavelength to be recorded. The epi- and endocardium are alternatively illuminated. The dyes then emit a fluorescent light assumed to be proportional to both the incident light and the transmembrane potential (TMP). Optical images are the surface recordings of this light by the cameras [3]. Images are recorded on the illuminated surface (reflexion mode) and on the opposite one (transillumination mode). For each time step, optical mapping hence produces four images.

The medium has a natural fluorescence (called background $F_{0}$ ) recorded when the tissue is at rest. Fluxes captured during an AP are denoted by $F$. The signal due only to the AP itself is $F-F_{0}$. We shall rather use the usual renormalization:

$$
g^{\star}=\overline{F_{0}} \max \left(0, \frac{F-F_{0}}{F_{0}}\right) \text {. }
$$

Indeed the $\max (\ldots)$ amounts to ignore negative, physically irrelevant, optical signals (due to noise). The multiplication by the average $\overline{F_{0}}$ of the background signal is a way to retrieve the correct amplitude of the signal. Our main goal is to reconstruct the $3 D$ front of the $A P$ from these $2 D$ optical data.

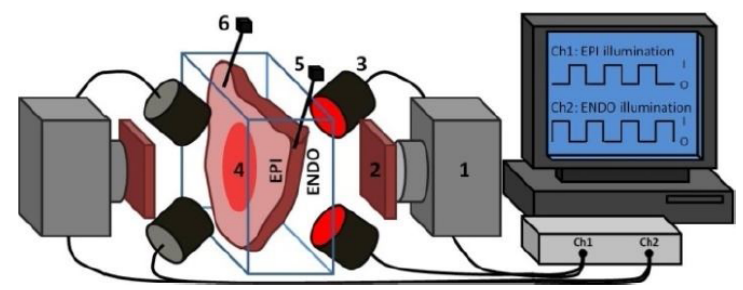

Fig. 1. The optical imaging setup: (1) CCD camera, (2) emission filter, (3) LED illumination, (4) tissue sample, (5) ECG electrode, (6) bipolar stimulating electrode.

\section{Model}

\subsection{Forward problem}

In order to write the mathematical model of these observations, we assume the following: the cameras record photon fluxes through the surfaces, the light interacts with the tissue material in the diffusive regime [2], and a Robin boundary condition can be used to model the interaction between the tissue and its environment. Hence the illumination light is described by its photon density $\phi_{0}$ that solves the diffusion equation

$$
\left\{\begin{array}{l}
-D_{0} \Delta \phi_{0}+\mu_{0} \phi_{0}=0 \quad \text { in } \Omega, \\
\phi_{0}+d_{0} \frac{\partial \phi_{0}}{\partial n}=0 \quad \text { on } \partial \Omega \backslash \Gamma, \quad \text { and } \phi_{0}=\frac{I_{0} \delta_{0}}{D_{0}} \quad \text { on } \Gamma,
\end{array}\right.
$$


where $\Omega \subset \mathbb{R}^{3}$ represents the slab of tissue, $\Gamma$ is the illuminated surface, and $n$ is the unit normal to $\partial \Omega$, outward of $\Omega$. The fluorescent light is assumed to be proportional to the TMP and the illumination light (multiplicative factor $\beta>0)$. Its photon density solves:

$$
\left\{\begin{array}{l}
-D \Delta \phi+\mu \phi=\beta\left(V_{m}-V_{0}\right) \phi_{0} \quad \text { in } \Omega, \\
\phi+d \frac{\partial \phi}{\partial n}=0 \quad \text { on } \partial \Omega .
\end{array}\right.
$$

In both equations, the optical parameters $D, \mu, d$ stand respectively for diffusion coefficient, absorption coefficient and extrapolation distance. The attenuation length is the parameter $\delta_{0}$ defined by $\delta_{0}=\sqrt{\frac{D_{0}}{\mu_{0}}}$. The intensity of the illumination, assumed uniform, is the parameter $I_{0}$. The multiplicative factor $\beta>0$ is known for the dye used during the experiments. The dyes are assumed to be uniformly distributed in the tissue. Finally the fluxes measured through the surfaces are given by Fick's law:

$$
g=-D \frac{\partial \phi}{\partial n} \quad \text { on the epi or endocardium. }
$$

Remark that the experimental flux $g^{\star}$ given by (1) does not satisfy equation (3), because of the renormalization. The quantity $F-F_{0}$ does. However we shall consider $g^{\star}$ as a good approximation of $g$, following the recommendations of the experimenters.

Since we consider a rectangular slab of tissue, we may have used structured meshes. We choose to work with unstructured meshes in order to allow more general geometries. This is necessary to study data from heart tissues. The diffusion equations are solved with with P1-Lagrange finite elements method using the solver FreeFem $++[4]$.

\subsection{Inverse problem}

The problem of retrieving the 3D spatial distribution of the TMP, denoted by $V_{m}(t, \mathbf{x})$ from the $2 \mathrm{D}$ optical signals at time $t>0$ is under-determined. Hereafter, $\mathbf{x}=(x, y, z)$ denotes a point in $\Omega$ with Cartesian coordinates $(x, y, z)$. Instead of finding the complete distribution $V_{m}(t, \mathbf{x})$, we look for a depolarization front at each time. Specifically, we assume that a surface $\mathcal{S}(t)=\{\mathbf{x} \in \Omega: f(t, \mathbf{x})=0\}$ defined as the level 0 of the function $f$ splits the domain $\Omega$ into the region $\Omega_{r}=\{\mathbf{x} \in \Omega: f(t, \mathbf{x})>0\}$ of tissue at rest, and the region $\Omega_{p}=\{\mathbf{x} \in \Omega:$ $f(t, \mathbf{x})<0\}$ of excited tissue. It follows that $V_{m}(t, \mathbf{x})=V_{p}$ if $\mathbf{x} \in \Omega_{p}$, and $V_{m}(t, \mathbf{x})=V_{0}$ if $\mathbf{x} \in \Omega_{r}$. We consider simple depolarization fronts $\mathcal{S}$ modeled

- either by the sphere centered in $\mathbf{x}_{0} \in \Omega$ and expanding with the velocity $c>0$ after the given time $t=t_{0} \geq 0$, defined by the level-set function $f(t, \mathbf{x})=\left|\mathbf{x}-\mathbf{x}_{0}\right|-c\left(t-t_{0}\right)$,

- or by the fixed ellipsoid centered in $\mathbf{x}_{0} \in \Omega$ and with radiuses $r_{x}, r_{y}, r_{z}>0$, defined by the level-set function $f(t, \mathbf{x})=\frac{\left(x-x_{0}\right)^{2}}{r_{x}^{2}}+\frac{\left(y-y_{0}\right)^{2}}{r_{y}^{2}}+\frac{\left(z-z_{0}\right)^{2}}{r_{z}^{2}}-1$. 
This level-set approach generalizes to more complex AP, once these simple cases are completely understood. In both cases, the inverse problem reduces to the identification of a small parameters set $\mathcal{P}=\left(\mathbf{x}, c, t_{0}\right) \subset \mathbb{R}^{5}$ (sphere) or $\mathcal{P}=\left(\mathbf{x}, r_{x}, r_{y}, r_{z}\right) \subset \mathbb{R}^{6}$ (ellipsoid). In order to identify these parameters, we minimize the least squares difference $e(\mathcal{P})$ between the actual measurements and the measurements computed from equations (2), (3), and (4) with a TMP as above:

$$
e(\mathcal{P})=\frac{1}{2} \sum_{i=1}^{4}\left\|g_{\mathcal{P}}^{i}-g^{\star, i}\right\|_{L^{2}\left(\mathbb{S}_{i}\right)}^{2},
$$

where the functions $g^{\star, i}$ are the data. Here $i$ refers to one of the four images ( $i \in$ $\{1,2,3,4\})$, and the surface $\mathbb{S}_{i}$ is either the epicardium or the endocardium, as detailed in Table 1 . Although this is the natural way to define the cost function,

\begin{tabular}{lll}
\hline$\#$ & illuminated surface & measured surface \\
\hline 1 & epicardium & $\mathbb{S}_{1}=$ epicardium \\
2 & epicardium & $\mathbb{S}_{2}=$ endocardium \\
3 & endocardium & $\mathbb{S}_{3}=$ endocardium \\
4 & endocardium & $\mathbb{S}_{4}=$ epicardium \\
\hline
\end{tabular}

Table 1. References of the measures

the value $I_{0}$ of the illumination in equation (2) is unknown, while the optical parameters are. Consequently, and since equations (2), (3), and (4) are linear, the density $\phi_{0}$, or $\phi$, can only be computed up to a multiplicative constant. The mapping $I_{0} \mapsto g^{i}$ is also linear, the measurement in-silico $g^{i}$ is consequently proportional to $I_{0}$, and we can change the cost function to account for this unknown value. A first idea is to identify the intensity $I_{0}$, and consider the following modified cost function:

$$
e(\mathcal{P})=\frac{1}{2} \sum_{i=1}^{2} \sum_{j=1}^{2}\left\|I_{0}^{i} g_{\mathcal{P}}^{i j}-g^{\star, i j}\right\|_{L^{2}\left(\mathbb{S}_{j}\right)}^{2},
$$

where $i, j \in\{1,2\}, i$ stands for the illuminated surface while $j$ stands for the measured surface $(i=j$ for the reflexion mode, and $i \neq j$ for the transillumination mode). Since the intensities are different, we have two additional parameters to retrieve, $I_{0}^{1}$ and $I_{0}^{2}$. A second idea consists in comparing the normalized fluxes:

$$
e(\mathcal{P})=\frac{1}{2} \sum_{i=1}^{4}\left\|\frac{g^{i}}{\left\|g^{i}\right\|}-\frac{g^{\star, i}}{\left\|g^{\star, i}\right\|}\right\|_{L^{2}\left(\mathbb{S}_{i}\right)}^{2},
$$

where the inner norms $\|\cdot\|$ are also $L^{2}$ norms on the surface $\mathbb{S}_{i}$. In this case there is no additional parameter to be identified, but the problem becomes nonlinear with respect to $V_{m}$.

In all cases, a fixed-step gradient method followed by the BFGS algorithm is used to solve the inverse problem. We need the gradient of all the cost functions $e$ 
with respect to the unknown parameters $\mathcal{P}$, which is computed with the adjoint method.

\section{Summary of the results and issues}

\subsection{Summary of previous results}

In this part we quickly recall the first results we obtained. We first compared the formula derived in [2] and our method for the expanding sphere and the cost function (5) on data in silico.

Figure 2 shows the depth of the source computed from Khait's formula (diamonds) and from our method (squares) as a function of time. Results are presented for inclusions at four different locations, excitation time $t_{0}=0$ and velocity $c=0.5 \mathrm{~m} . \mathrm{s}^{-1}$. The last example was carried out on a cylinder, in order to illustrate the case of a complex geometry. For all cases we retrieved the complete
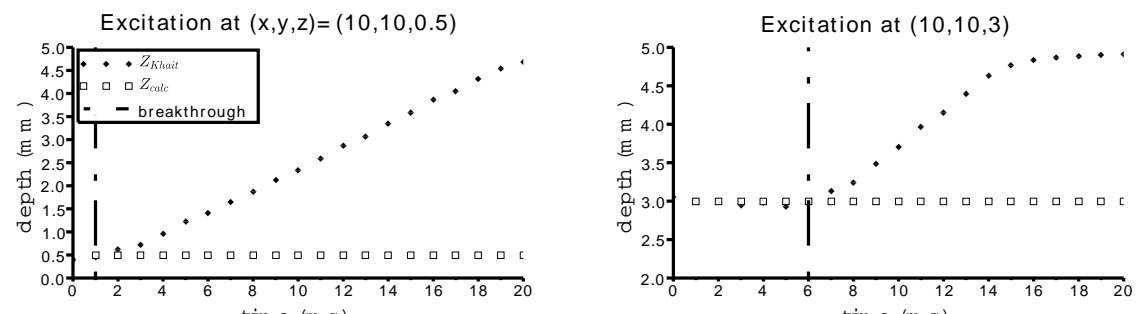

Excitation at êt (ms $s, 9,9.5)$
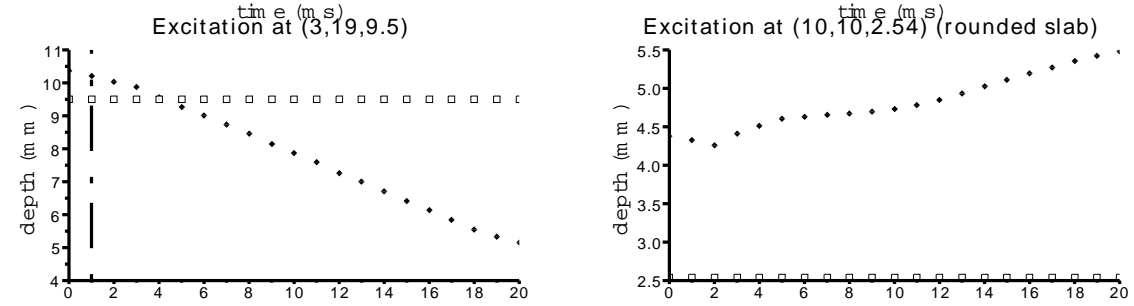

Fig. 2. Comparison between with Khait's formula (diamonds) and our method (squares). The vertical lines mark the breakthrough of the wave on the observed surface.

unknown location $\mathbf{x}_{0}$ of the source, at any time, and even after the breakthrough, with an accuracy up to machine precision. Additionally, the velocity $c$ could be recovered from any time-sequence of data. These were very good results, based on data in-silico.

The method was then tested on the experimental data from the optical phantom experiments set up by Khait and coworkers [2]. In this case, fixed ellipsoidal sources were considered and the intensity $I_{0}$ of the illumination was unknown. We chose to work with the normalized cost function (7), so that we had to identify six parameters.

Figure 3 shows the results obtained for a phantom located at a depth $z_{0}=13$ $\mathrm{mm}$. Although the reconstruction of the photon fluxes looks qualitatively correct 
(first and second rows of images), the reconstructed depth is $z^{\star}=16.7 \mathrm{~mm}$. In order to understand this large error (3 $\mathrm{mm}$ over a total depth of $20 \mathrm{~mm}$ ),

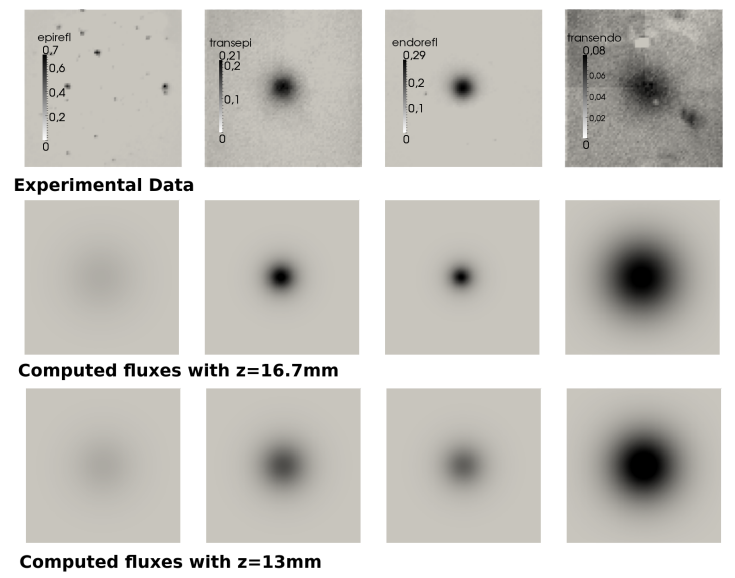

Fig. 3. Results for one set of experimental data. Domain size: 40x40x20mm. Columns 1 and 2: records on the epicardium. Columns 3 and 4: records on the endocardium.

we computed the theoretical observations associated to the exact experimental location of phantom source: from $\mathcal{P}$ known, we compute the TMP distribution $V_{m}(\mathbf{x})$, solve equations (3) and (4), and finally compute the observation $g$ with equation (4). These normalized fluxes (third row of images on figure 3) clearly have different amplitudes than the experimental signal. These fluxes are also more diffuse than the experimental ones. We observe the same behavior with seven other experimental phantoms. We tried to replace the measure $g^{\star}$ (eq. (1)) by the simpler difference $\max \left(F-F_{0}, 0\right)$ and obtained similar errors on the location. We deduce from these results that the optical model (2) and (3) is questionable. In the next section, we identify and study in depth several possible sources of error.

\subsection{Model improvements to fix the mismatch}

Uniform Illumination. First, we addressed the approximation of uniform distribution of the illumination $I_{0}$. We tested the effect of several spatially distributed illumination intensities $I_{0}(x, y)$ : constant, narrow Gaussian, diffuse Gaussian, supposed to mimic the experimental lights. They read

$$
I_{0}(x, y)=A \exp \left[-\left(\frac{\left(x-x_{0}\right)^{2}}{\sigma_{x}^{2}}+\frac{\left(y-y_{0}\right)^{2}}{\sigma_{y}^{2}}+\frac{\left(x-x_{0}\right)\left(y-y_{0}\right)}{2 \sigma_{x y}}\right)\right] .
$$

We observed the same fluxes $g$ for the three cases. Indeed, the source term $\left(V_{m}(\mathbf{x})-V_{0}\right) \phi_{0}(\mathbf{x})$ in the fluorescence equation is the same in all cases because the photon density $\phi_{0}$ becomes quickly constant inside the tissue (see Figure 4). Finally it is not a limitation to consider an uniform illumination. 


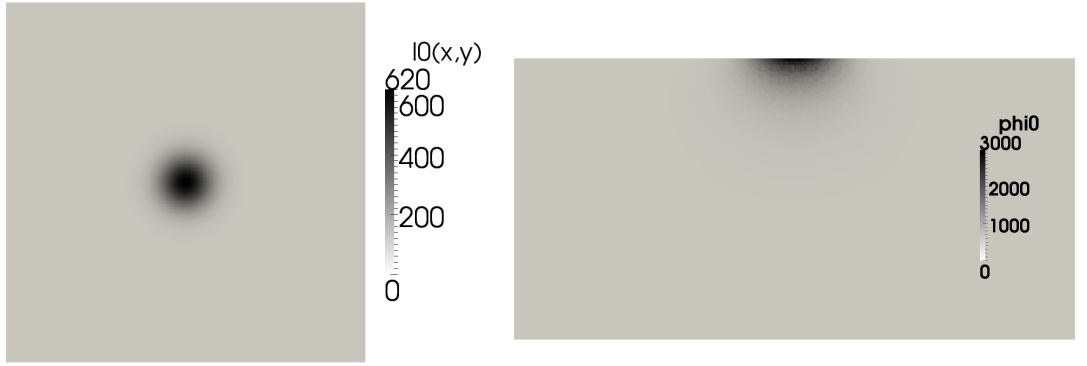

Fig. 4. Narrow Gaussian example for an epi-illumination. Domain size: 40x40x20mm. On the left: spatial distribution of $I_{0}$, on the right: photon density $\phi_{0}$, cut plane in $z$-direction.

Optical parameters. Another possibility is that the values of the optical parameters are not correct. In order to understand the role of these parameters, we derive a dimensionless version of the equations. Consider a space scale $L>0$ and let rescale $\mathbf{x}$ as : $\mathbf{x}^{\prime}=\frac{\mathbf{x}}{L}$. Equations (2) and (3) rewritten

$$
\left\{\begin{array} { l } 
{ - \frac { D _ { 0 } } { L ^ { 2 } } \Delta \phi _ { 0 } + \mu _ { 0 } \phi _ { 0 } = 0 \quad \text { in } \Omega , } \\
{ \phi _ { 0 } + \frac { d _ { 0 } } { L } \frac { \partial \phi _ { 0 } } { \partial n } = 0 \quad \text { on } \partial \Omega \backslash \Gamma , } \\
{ \phi _ { 0 } = \frac { I _ { 0 } \delta _ { 0 } } { D _ { 0 } } \quad \text { on } \Gamma , }
\end{array} \quad \left\{\begin{array}{l}
-\frac{D}{L^{2}} \Delta \phi+\mu \phi=\beta\left(V_{m}-V_{0}\right) \phi_{0} \quad \text { in } \Omega, \\
\phi+\frac{d}{L} \frac{\partial \phi}{\partial n}=0 \quad \text { on } \partial \Omega .
\end{array}\right.\right.
$$

We define $\gamma=\frac{I_{0} \delta_{0}}{D_{0}}$ and rescale the density as $\phi_{0}^{\prime}=\frac{\phi_{0}}{\gamma}$ and $\phi^{\prime}=\frac{\phi}{\gamma}$, in such a way that:

$$
\left\{\begin{array} { l } 
{ - \frac { D _ { 0 } } { L ^ { 2 } } \Delta \phi _ { 0 } ^ { \prime } + \mu _ { 0 } \phi _ { 0 } ^ { \prime } = 0 \quad \text { in } \Omega , } \\
{ \phi _ { 0 } ^ { \prime } + \frac { d _ { 0 } } { L } \frac { \partial \phi _ { 0 } ^ { \prime } } { \partial n } = 0 \quad \text { on } \partial \Omega \backslash \Gamma , } \\
{ \phi _ { 0 } ^ { \prime } = 1 \text { on } \Gamma , }
\end{array} \quad \left\{\begin{array}{l}
-\frac{D}{L^{2}} \Delta \phi^{\prime}+\mu \phi^{\prime}=\beta\left(V_{m}-V_{0}\right) \phi_{0}^{\prime} \quad \text { in } \Omega, \\
\phi^{\prime}+\frac{d}{L} \frac{\partial \phi^{\prime}}{\partial n}=0 \quad \text { on } \partial \Omega .
\end{array}\right.\right.
$$

Dividing the diffusion equation by $\mu_{0}$ (resp. $\mu$ ) the dimensionless system reads:

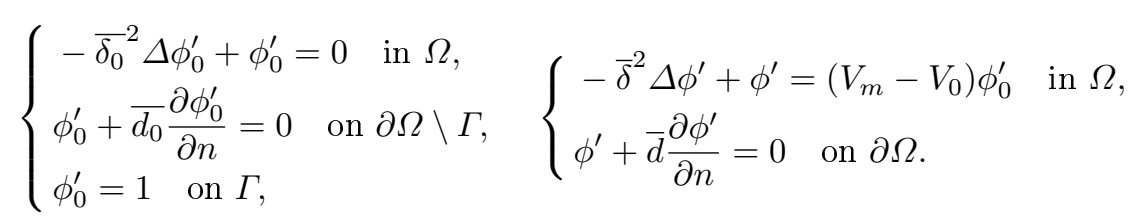

where ${\overline{\delta_{0}}}^{2}=\frac{D_{0}}{\mu_{0} L^{2}}, \overline{d_{0}}=\frac{d_{0}}{L}, \bar{\delta}^{2}=\frac{D}{\mu L^{2}}$ and $\bar{d}=\frac{d}{L}$ are dimensionless optical parameters. Finally the fluxes are given by $g^{\prime}=\phi^{\prime}$ on the epi or endocardium. The other parameters, $I_{0}$ and $\beta$ are hidden in a dimensionless number $\gamma$ and we compare the experimental fluxes $g^{\star}$ to $\gamma g^{\prime}$. The optical system is characterized by the four optical parameters. Adding the two terms $\gamma$ (one for each illumination), 
we end up with six parameters. We tried to solve a second inverse problem: knowing the characteristics $\mathcal{P}$ of the inclusion, identify the six new parameters by minimizing the cost function

$$
e_{1}\left({\overline{\delta_{0}}}^{2}, \overline{d_{0}}, \bar{\delta}^{2}, \bar{d}, \gamma^{1}, \gamma^{2}\right)=\frac{1}{2} \sum_{i=1}^{2} \sum_{j=1}^{2}\left\|\gamma^{i} g^{i j j}-g^{\star, i j}\right\|_{L^{2}\left(\mathbb{S}_{j}\right)}^{2} .
$$

To date, the numerical solutions to this optimization problem are still being computed.

Other possibilities. In our model we do not consider the distance between the preparation and the camera. We could consider the diffusion of the photon density in the air by ensuring the continuity of the fluxes at the border medium/air. Instead, we impose a Robin condition on all the surfaces. We consider that the fluxes are recorded directly through the tissue surfaces, and not through the Plexiglas, because the Plexiglas has a negligible absorption coefficient.

\section{Discussion}

Modeling the optical measurements by the diffusion equations (2) and (3) is widely used in cardiac optical mapping. But when we confront the measurements obtained with this model to the experimental ones in a well controlled setup, we observe an important mismatch (fig. 3). We studied several ideas that might explain the differences, always with a negative result. To our opinion, this is likely to suggest that the diffusive regime is a too coarse approximation of the interaction between light and matter in the cardiac context. If this is confirmed by further experiments, the complete radiative transfer equation (RTE) might be used to model the measurements.

\section{Conclusion}

The aim of this study was to detail our investigations concerning the current model of cardiac optical mapping measurements. Having recalled previous results we described our attempts to improve our mathematical model. The most likely assumption was that the illumination on the tissue was not constant. Few tests showed that it was not the key. We eliminated several other sources of error, but there remains some more. We keep on working on the dimensionless problem in order to identify its parameters. Otherwise the RTE might be used.

\section{Acknowledgments}

This work is partially supported by the grant number ANR-10-IAHU-04 from the French government, and by the Conseil Régional Aquitaine. 


\section{References}

1. D. S. Rosenbaum, J. Jalife: Optical mapping of cardiac excitation and arrhythmias. Wiley-Blackwell (2001)

2. V. D. Khait, O Bernus, S. F. Mironov, A. M. Pertsov: Method for 3-dimensional localization of intramyocardial excitation centers using optical imaging. Journal of Biomedical Optics 11 (2006)

3. R. D. Walton, C. D. Lawrence-Xavier, I. Tachtsidis, O. Bernus: Experimental validation of alternating transillumination for imaging intramural wave propagation. IEEE, 1676-1679 (2011)

4. F. Hecht: New development in FreeFem ++ . Journal of Numerical Mathematics 20, 251-265 (2012)

5. M.J. Bishop, B. Rodriguez, J. Eason, J.P. Whiteley, N. Trayanova, D.J. Gavaghan: Synthesis of voltage-sensitive optical signals: application to panoramic optical mapping. Biophys J 90(8):2938-45 (2006) 